\title{
Other journals in brief
}

\section{HELICOBACTER PYLORI}

\section{Yeast of the oral cavity is the reservoir for Heli(c)obacter pylori}

Salmanian A-H, Siavoshi F et al. J Oral Pathol Med 2008; 37: 324-328

\section{Oral cavity and Helicobacter pylori.}

The aim of this study was to explore an important earlier postulate made by these authors, that there is an intimate relationship between Helicobacter pylori and oral yeasts. DNA was extracted from 13 different strains of Candida albicans containing, on microscopic examination, bacterium-like bodies suggestive of $H$. pylori. Fragments of these genes, specific to $H$. pylori, were then amplified using polymerase chain reaction. There was a 98\% homology between these specific genes, extracted from the $C$. albicans and the $H$. pylori positive control genes. The authors conclude that the endosymbiotic relationship between H. pylori and C. albicans could explain the re-inoculation of the stomach by $H$. pylori after antimicrobial therapy.

DOI: 10.1038/sj.bdj.2008.1076

\section{BRUXISM}

\section{Review article: principles of the management} of bruxism

Lobbezoo F, van der Zaag J et al. J Oral Rehabil 2008; 35: 509-523

\section{Bruxism is best managed using the triple-P approach.}

Despite 'at least $\$ 1$ billion per year' spent in the USA on the fabrication of mouth guards, the authors were only able to identify less than 20 randomised control studies investigating all treatment approaches for bruxism. During the past decade, well-designed studies using pharmacological approaches have been carried out. The drugs include muscle relaxants and other agents that have effects at the neuromuscular junction. This current strategy has replaced behavioural methods such as group therapy using habit retraining. With regard to occlusal approaches the authors state that there is '...no support in the literature for... equilibration, rehabilitation and orthodontic alignment for the management of bruxism'. In addition, there is no evidence 'for the long term efficacy or safety' of the new chair-side fabricated appliances such as NTI. The authors conclude, 'in the absence of definitive evidence, bruxism is best managed using the triple-P approach - plates, pep talks and pills'.

\section{HISTORICAL ORAL MEDICINE}

\author{
Nikolskiy's sign revisited \\ Juneja M. J OralSci2008; 50: 213-214
}

\section{Nikolskiy's sign or phenomenon - now an irrelevance that causes damage.}

The diligent student fathomed out that Nikolskiy's sign, the historical diagnostic indicator for pemphigus, has modifications. This short communication, which reads like a Russian romantic novel, states that the original sign, first described in 1894 by Nikolskiy, comprised three variants, with the common component of the physician removing/dislodging the horny layer from the diseased mucosa. Wilhelm Lutz, in 1957, then qualified this clinical finding by stating 'if (one) carefully rubbed on an uninvolved area .... blister will form' and called this the Nikolskiy phenomenon. To add to the confusion, there is the Asboe-Hansen sign which is similar to that described by Luntz but is diagnostic of one of several vesicular-bullous conditions. The author does not mention the well established standard diagnostic method for pemphigus of direct immunofluoresence. DOI: 10.1038/sj.bdj.2008.1078

\section{ETHNICITY AND PERIODONTAL DISEASE}

\section{Race/ethnicity and inflammation - is there a link to periodontal disease?}

Albert M A. J Periodonto/ 2008; 79: 1121-1123

\section{Research priorities.}

When compared to others, black people are associated with twice the prevalence of periodontal disease. Elevated levels of C-reactive protein (CRP) are associated with body mass index, hypertension, hypercholesterolemia, socioeconomic status, diabetes and smoking. However, when taking these into account, there are still unexplained differences in CRP between black women and those from other ethnic backgrounds. In addition, 'regardless of socioeconomic status (SES), black women in the United States have the highest infant mortality rates'. What then are the research priorities? Are 'elevated CRP levels...due to race/ethnicity, periodontal disease, pregnancy or other factors'? Does 'the combination of periodontal disease and pregnancy...influence mother and child health outcomes'? And finally, does the treatment of periodontal disease, result in more favourable outcomes for heart disease and other pregnancy-associated conditions? DOI: 10.1038/sj.bdj.2008.1079 